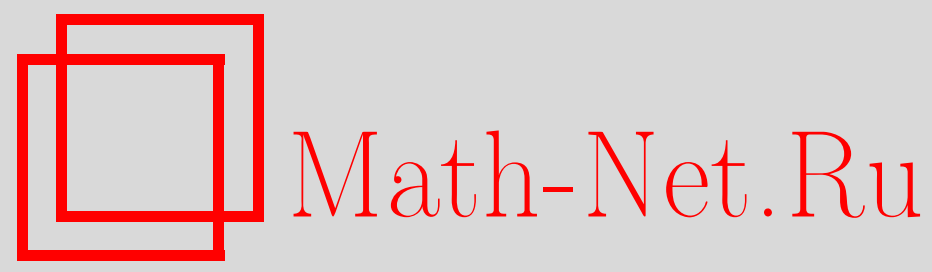

В. В. Римацкий, Построение явного базиса для допустимых правил вывода модальных логик, расширяющих $S 4.1$, Дискрет. матем., 2011, том 23, выпуск 4, 48-65

DOI: https://doi.org/10.4213/dm1161

Использование Общероссийского математического портала Math-Net.Ru подразумевает, что вы прочитали и согласны с пользовательским соглашением http://www . mathnet.ru/rus/agreement

Параметры загрузки:

IP : 54.172 .240 .79

26 апреля 2023 г., $18: 27: 03$ 


\title{
Построение явного базиса для допустимых правил вывода модальных логик, расширяющих $S 4.1$
}

\author{
(c) 2011 г. В. В. Римацкий
}

В статье исследуются базисы для допустимых правил вывода модальных логик. Известно, что большинство базовых нестандартных логик не имеют конечного базиса для допустимых правил. В представленной работе описывается явный базис для допустимых правил логик $S 4.1, G r z$ и их расширений, число которых по крайней мере счетно.

Полученный базис состоит из бесконечной последовательности правил, которые имеют компактную и простую форму. В случае конечности ширины логики базис для допустимых правил также состоит из конечной последовательности правил.

\section{1. Введение}

Современные приложения логики в компьютерных науках и в искусственном интеллекте нуждаются в языке, приспособленном для описания различных динамических систем. Язык неклассических логик (например, модальных или временных) успешно выполняет эту функцию. Но изначально факты, утверждения в этом языке описываются с помощью формул, которые предназначены для описания моделей в общем и не способны выразить изменяющиеся условия и предпосылки. Эти условия и предпосылки могут моделироваться с помощью различных вариантов понятия логического следования. Поэтому проблема адекватного моделирования логического следования является одной из важнейших проблем современной математической логики и математики в целом. Одна из важнейших особенностей нашего подхода к изучению логического следования состоит в том, что мы исследуем логическое следование в терминах правил вывода, секвентов, а не просто формул или утверждений.

Формализм описания свойств моделей посредством формул глубоко развит, широко распространен и подробно представлен в научной литературе. Он является базисом представления и изучения человеческого мышления. Однако, как уже было замечено, формулы описывают только стабильные, статичные явления; утверждение только фиксирует факт и не способно ухватить меняющиеся условия. Поэтому изучение (структурных) правил вывода (или секвентов) - выражений, имеющих посылки (заданный набор предположений) и заключение, - предоставляет нам более тонкий и выразительный аппарат для моделирования мышления и вычислений. Посылки правила вывода выражают текущую, заданную информацию как предположения, а заключение представляет вывод или факт, который мы можем получить из наших предположений. Правила вывода позволяют нам 
также моделировать стандартную ситуацию в изучении логического следования: даны некоторые предположения или факты, что из них следует, что является непротиворечивым следствием наблюдаемых фактов?

Очевидно, что понятие правила вывода обобщает понятие формулы: любая формула может быть рассмотрена как структурное правило вывода без посылки, без предположений. Однако, допустимые правила вывода оказались намного сильнее обычных структурных правил: благодаря примеру Р. Харропа (1960г., [2]) мы знаем, что даже интуиционистская логика Int не является структурно полной, то есть в ней существуют допустимые, но не выводимые правила вывода, правила, не представимые посредством формул. Благодаря примерам Г. Минца [3] и Дж. Порта [4] это также справедливо и для широкого класса модальных логик.

Понятие (структурного) допустимого правила вывода было впервые введено Лоренценом [5] в 1955 г. Для произвольной логики допустимыми являются те правила вывода, которые не изменяют множество доказуемых теорем данной логики. Понятно, что любое выводимое правило является допустимым в заданной логике, но обратное в общем случае не верно, как показывают упомянутые выше примеры Р. Харропа, Г. Минца и Дж. Порта. Непосредственно из определения можно также заключить, что множество всех допустимых в логике $\lambda$ правил вывода образует наибольший класс правил вывода, которыми мы можем расширить аксиоматическую систему данной логики, не изменяя множество доказуемых теорем. Кроме того, допустимые правила значительно усиливают дедуктивную систему заданной логики. Известно, что выводимые правила вывода могут заменить в доказательстве некоторый фрагмент фиксированной длины, то есть сократить доказательство линейно. Допустимые правила, не выводимые в данной логике, могут сократить доказательство более существенно.

Начало истории изучения допустимых правил может быть датировано 1975 г. с появления проблемы Х. Фридмана [6] о существования алгоритмического критерия допустимости правил в интуиционистской логике Int. В классической логике вопрос допустимости решался тривиально - допустимы только выводимые, доказуемые правила. В случае неклассических логик примеры Харропа и Минца, а позже Порта, показали, что существуют допустимые, но не доказуемые правила вывода. В середине 70-х Г. Минц [3] получил достаточные условия выводимости правил специальной формы. Положительное решение проблемы Фридмана о существовании алгоритма, распознающего допустимость правил вывода в интуиционистской логике Int, было получено В. В. Рыбаковым в 1984 г. [7].

Для широкого класса модальных и суперинтуиционистских логик критерий допустимости правил вывода был позднее сформулирован в [1]. Позднее Гиларди [8] нашел другое решение для логики Int с помощью проективных формул.

К проблеме А. Кузнецова (1973) о существовании конечного базиса для допустимых правил вывода логики Int восходит другой способ описания всех допустимых правил логики. Ведь имея базис для допустимых правил, все остальные выводятся из него как следствия. Первый положительный результат в изучении базисов для допустимых правил вывода был получен А. Циткиным [9], который нашел базис для всех допустимых в Int квазихарактеристических правил вывода. Изначально исследование базисов для допустимых правил вывода нестандартных логик фокусировалось на наиболее важных индивидуальных логиках, таких как логика доказуемости $G L$, или системах $S 4, S 5$; а также на обобщении существующих методов и получении общей техники, применимой не только к отдельным, индивидуальным логикам, а к целым обширным подклассам логик, включающих наиболее интересные и важные логики. В общем проблема Кузнецова о существовании конечного базиса для допустимых правил вывода решалась отрицательно не только для Int (Рыбаков, [10]), но и для большинства других базовых логик. В. В. Рыбаков 
(гл. 4 в [1]) показал, что логики Int, $K C, K 4, S 4, G r z$ и многие другие не имеют конечного базиса для допустимых правил от конечного числа переменных.

Учитывая отрицательное решение проблемы Кузнецова для многих базовых неклассических логик, к концу 20-го века базис для допустимых правил вывода мог быть получен только из известного алгоритмического критерия допустимости (см. гл. 3.5 в [1]). Однако, этот критерий является вычислительно сложным и неприменимым для описания такого базиса в легко обозримой форме. Поэтому становится актуальной проблема явного описания легко обозримого базиса для всех допустимых правил вывода хотя бы для основных базовых логик, а также для тех «сильных» табличных логик, которые имеют конечный базис допустимых правил (см. $[11,12])$. Первый шаг в этом направлении был сделан в 2000 г.: в статье [13] был получен рекурсивный базис для допустимых правил интуиционистской логики Int, состоящий из правил в полуредуцированной форме. Позже Р. Иемхофф $[14,15]$ получила явный базис допустимых правил логики Int. В статье [16] В. В. Рыбаков построил точный базис для всех допустимых правил логики $S 4$.

Заметим, что практически во всех упомянутых выше результатах существенно используется транзитивность бинарного отношения на фреймах. Только в последние годы стали появляться результаты, посвященные правилам вывода нетранзитивных и многомодальных логик. Проблема допустимости в минимальной модальной логике $K$ и многих других нетранзитивных или многомодальных логиках по-прежнему остается открытой. Автору известно относительно немного результатов, посвященных исследованию допустимых правил вывода в таких логиках. Относительно недавно Волтер и Захарьящев [17] показали, что модальные логики с дополнительной универсальной модальностью, расположенные между $K u$ и $K 4 u$, неразрешимы по допустимости. В [18] положительно решена проблема допустимости в интранзитивной мономодальной логике с оператором «завтра». Проблеме разрешимости по допустимости различных временных логик посвящены $[19,20,21]$. В [22, 23] исследуется проблема допустимости правил вывода многомодальных логик.

В представленной работе описывается явный базис для допустимых правил целого класса (по крайней мере счетного) финитно аппроксимируемых логик, расширяющих логику $S 4.1$ и удовлетворяющих некоторым дополнительным условиям. Полученный базис имеет компактную и наглядную форму. В случае конечности ширины заданной логики мы показываем, что базис для допустимых правил данной логики также конечен.

\section{2. Определения, предварительные результаты}

Вначале напомним кратко необходимые определения и результаты (для детального знакомства с предметом рекомендуем [1]). Далее мы рассматриваем только логики, расширяющие $S 4$, поэтому все фреймы рефлексивны и транзитивны.

Язык модальных логик состоит из счетного множества пропозициональных переменных $p_{1}, \ldots, p_{n}, \ldots$, логических связок классической логики $\neg, \wedge, \vee, \rightarrow$ и унарного модального оператора $\square$. Нормальная модальная логика есть множество модальных формул $L$, содержащее все пропозициональные тавтологии, схему аксиом $\square(\alpha \rightarrow \beta) \rightarrow$ $(\square \alpha \rightarrow \square \beta)$, и замкнутое относительно подстановок, правила отделения $\alpha, \alpha \rightarrow \beta \vdash \beta$ и необходимости $\alpha \vdash \square \alpha$. Минимальная модальная логика обозначается как логика $K$. Расширение логики $K$ схемой $\square \alpha \rightarrow \square \alpha$ обозначается $K 4$; расширение $K 4$ схемой $\square \alpha \rightarrow \alpha$ порождает логику $S 4$. Если $L-$ нормальная модальная логика, то для формул $\alpha \in L$ пишем $\vdash_{L} \alpha$ или $L \vdash \alpha$. Если логика $L$ фиксирована или ясна из контекста, то ее обозначаем для простоты $\vdash \alpha$. 
Фрейм $\mathscr{F}:=\langle F, R\rangle$ есть пара, где $F$ - непустое множество и $R$ - бинарное отношение на $F$. Содержательно $W$ представляет множество всех «возможных» миров, $R-$ отношение перехода из одного мира в другой. Далее базисное множество и сам фрейм далее будем обозначать одной и той же буквой.

Моделью называем тройку $\mathcal{M}:=\langle F, R, V\rangle$, где $\langle F, R\rangle$ - фрейм, и означивание $V$ есть отображение множества пропозициональных переменных в множество $2^{F}$ всех подмножеств множества $F$. Означивание $V$ ставит в соответствие каждой переменной множество миров $V(p)$, в которых переменная $p$ истинна. Обозначим истинность переменной $p$ в точке $x \in F$ при заданном означивании $V$ как $(F, x) \vDash_{V} p$. В тех случаях, когда базисное множество (фрейм) ясно из контекста, истинность переменной будем записывать как $x \vDash_{V} p$.

Истинность формулы $\alpha$ в точке $x \in F$ при заданном означивании $V$ индуктивно определяется следующим образом:

$$
\begin{aligned}
x \vDash_{V} p & \Longleftrightarrow x \in V(p) ; \\
x \vDash_{V} \neg \alpha & \Longleftrightarrow x \vDash_{V} \alpha ; \\
x \vDash_{V} \alpha \vee \beta & \Longleftrightarrow x \vDash_{V} \alpha \text { или } x \vDash_{V} \beta ; \\
x \vDash_{V} \alpha \wedge \beta & \Longleftrightarrow x \vDash_{V} \alpha \text { и } x \vDash_{V} \beta ; \\
x \vDash_{V} \alpha \rightarrow \beta & \Longleftrightarrow x \vDash_{V} \alpha \text { или } x \vDash_{V} \beta ; \\
x \vDash_{V} \square \alpha & \Longleftrightarrow \forall y \in F\left(x R y \Rightarrow y \vDash_{V} \alpha\right) ; \\
x \vDash_{V} \diamond \alpha & \Longleftrightarrow \exists y \in F\left(x R y \& y \vDash_{V} \alpha\right) .
\end{aligned}
$$

Формула $A$ истинна на модели $\mathcal{M}=\langle F, R, V\rangle$ (обозначение $\mathcal{M} \vDash A$ или $F \vDash_{V} A$ ), если данная формула $A$ истинна на каждом элементе модели $\mathcal{M}$ при означивании $V$. Формула $A$ истинна на фрейме $F$ (обозначение $F \vDash A$ ), если она истинна на любой модели $M$, порожденной $F$, то есть истинна при любом означивании на $F$.

Подмножество $\mathscr{X}$ заданной модели $\mathcal{M}$ называется формульным (определимым), если существует формула $\alpha$ такая, что $\forall z \in M\left[z \vDash_{V} \alpha \Longleftrightarrow z \in \mathscr{X}\right]$. Соответственно, элемент $z \in \mathcal{M}$ является формульным, если множество $\{z\}$ формульное. Означивание $V$ определимо (формульное) в модели $\mathcal{M}$, если для любой переменной $p$ из области $V$, множество $V(p)$ формульное.

Модель $M=\langle F, R, V\rangle$ называется адекватной для логики $L$ ( $L$-моделью), если любая формула, доказуемая в логике $L$, истинна на данной модели. Соответственно, фрейм $\langle F, R\rangle$ адекватен для логики $L$, если на нем истинны все доказуемые формулы логики $L$. Класс фреймов $K$ называется характеристическим для логики $L$, если любой фрейм из данного класса адекватен для $L$ и для любой формулы, не доказуемой в $L$, найдется фрейм из класса $K$, на котором опровергается данная формула. Для заданного класса фреймов $\mathscr{K}$, логика $L(\mathscr{K})$, порожденная $\mathscr{K}$, есть множество всех формул, истинных на всех фреймах из $\mathscr{K}$. В данном случае говорим, что логика $L(\mathscr{K})$ порождена классом фреймов $\mathscr{K}$.

Модальная логика $L$ называется разрешимой, если для любой формулы существует алгоритм, позволяющий установить ее доказуемость в данной логике. Модальная логика $L$ называется финитно аппроксимируемой, если для любой формулы $\alpha$, не доказуемой в $L$, существует конечный фрейм (или конечная алгебра), адекватный $L$, на котором не истинна формула $\alpha$. Модальная логика $L$ обладает дизъюнктивным свойством, если для любых формул $\alpha, \beta$ из доказуемости в $L$ формулы $\square \alpha \vee \square \beta$ следует доказуемость в $L$ одной из формул $\square \alpha$ или $\square \beta$.

Напомним, что если $\langle W, R\rangle-$ некоторый фрейм, то множество $C \subseteq W$ называется сгустком, если 
(1) для любых $x, y$ из $C$ выполняется $x R y$;

(2) для любых $x \in C$ и $y \in W(x R y \& y R x) \Longrightarrow y \in C$.

Сгусток называется собственным, если $|C|>1$; в противном случае - одноэлементным или вырожденным. Для элемента $a \in F$ через $C(a)$ обозначим сгусток, порожденный элементом $a$.

Любое множество попарно несравнимых по отношению $R$ сгустков фрейма $F$ называется антицепью. Антицепь $\mathscr{A}$ называется нетривиальной, если $\mathscr{A}$ состоит по крайней мере из двух различных сгустков, в противном случае она называется тривиальной. Для любого элемента $a \in F$ обозначим

$$
a^{R}=\{z \mid a R z\}, \quad a^{<R}=a^{R} \backslash C(a),
$$

и будем говорить, что элемент $a$ порождает как корень подфрейм $a^{R}$ фрейма $F$. Фрейм $\mathscr{F}$ - корневой, если существует элемент $a \in \mathscr{F}$ такой, что $\forall b \in \mathscr{F} a R b$. Данный элемент $a$ называем также корнем $\mathscr{F}$. Множество

$$
X^{R}:=\bigcup\left\{z^{R} \mid z \in X\right\}
$$

называется открытым подфреймом, порожденным $X$. Понятия корневой модели, подмодели и открытой подмодели определяются аналогичным образом.

Сгусток $C(a)$ из $F$ есть ко-накрытие для множества (или антицепи) $X \subseteq F$, если $a^{R} \backslash C(a)=X^{R}$. Говорим, что элемент $a$ есть ко-накрытие для $X \subseteq F$, если одноэлементный сгусток $C(a)$ образует ко-накрытие для $X$. Под ко-накрытием далее понимаем одноэлементный сгусток, являющийся ко-накрытием.

Ко-накрытие, порождающее как корень $L$-фрейм, называем $L$-ко-накрытием.

Говорим, что фрейм $\mathscr{F}$ является $L$-фреймом, если все теоремы логики $L$ истинны на $\mathscr{F}$ при любом означивании переменных. Соответственно, $L(\mathscr{F})-$ множество формул, истинных на $\mathscr{F}$, есть логика, порожденная фреймом $\mathscr{F}$.

Глубиной элемента $z$ модели (фрейма) F्F называется максимальное число сгустков в цепях сгустков, начинающихся со сгустка, содержащего $z$. Множество всех элементов фрейма (модели) $\mathscr{F}$ глубины не более чем $n$ будем обозначать $S_{\leqslant n}(\mathscr{F})$, а множество элементов глубины $n$ обозначим $S_{n}(\mathscr{F})$.

Для заданного фрейма $\mathscr{F}$, заданного означивания $V$ и правила вывода

$$
r:=\left\{\alpha_{1}, \ldots, \alpha_{k} / \beta\right\}
$$

говорим что $r$ истинно на $\mathscr{F}$ при означивании $V$ (обозначаем $\mathscr{F} \vDash_{V} r$ ), если как только $\forall z \in \mathscr{F} \forall i\left(z \vDash_{V} \alpha_{i}\right)$, то $\forall z \in \mathscr{F}\left(z \vDash_{V} \beta\right)$. Правило $r$ истинно на $\mathscr{F}$, если $r$ истинно на $\mathscr{F}$ при любом означивании $V$ (обозначаем $\mathscr{F} \models r$ ). Аналогично определяется истинность правила на заданной модели: $r$ истинно на $\mathcal{M}$, если как только $\forall z \in \mathcal{M} \forall i\left(z \vDash_{V} \alpha_{i}\right)$, то $\forall z \in M\left(z \vDash_{V} \beta\right)$.

Правило вывода $\alpha_{1}\left(p_{1}, \ldots, p_{n}\right), \ldots, \alpha_{k}\left(p_{1}, \ldots, p_{n}\right) / \beta\left(p_{1}, \ldots, p_{n}\right)$ называется допустимым в логике $L$ (обозначаем $r \in \operatorname{Ad}(L)$ ), если для любых формул $\delta_{1}, \ldots, \delta_{n}$ из $\left(\forall j \alpha_{j}\left(\delta_{1}, \ldots, \delta_{n}\right) \in L\right)$ следует $\beta\left(\delta_{1}, \ldots, \delta_{n}\right) \in L$.

Допустимые правила пропозициональной модальной (суперинтуиционистской) логики $L$ имеют алгебраическое описание - им соответствуют квазитождества, истинные на свободной алгебре счетного ранга $\mathfrak{F} w(L)$ многообразия алгебр $\operatorname{Var}(L)$, соответствующего данной логике, то есть справедливо следующее утверждение (см. гл. 3 в [1]). 
Предложение 1. Правило вывода

$$
r=\left\{\alpha_{1}\left(p_{1}, \ldots, p_{n}\right), \ldots, \alpha_{k}\left(p_{1}, \ldots, p_{n}\right) \beta\left(p_{1}, \ldots, p_{n}\right)\right\}
$$

допустимо в логике $L$, если и только если на свободной алгебре счетного ранга $\mathfrak{F}_{w}(L)$ из многообразия алгебр $\operatorname{Var}(L)$ истинно квазитождество

$$
r^{*}=\left\{\alpha_{1}\left(p_{1}, \ldots, p_{n}\right)=1 \& \ldots \& \alpha_{k}\left(p_{1}, \ldots, p_{n}\right)=1 \Longrightarrow \beta\left(p_{1}, \ldots, p_{n}\right)=1\right\} .
$$

Правило $r$ называется следствием правил $r_{1}, \ldots, r_{k}$ в логике $L$, если заключение $r$ выводимо из посылок $r$ с помощью теорем $L$, правил $r_{1}, \ldots, r_{k}$ и постулированных правил вывода $L$. Множество $\operatorname{Ad}^{*}(L)$ допустимых правил логики $L$ называем базисом допустимых правил, если для любого допустимого правила $r$ найдутся правила $r_{1}, \ldots, r_{k} \in \mathrm{Ad}^{*}(L)$ такие, что $r$ выводимо из $r_{1}, \ldots, r_{k}$ в логике $L$.

Предложение 2 ([1]). Множество $r_{1}, \ldots, r_{k}$ есть базис допустимых правил вывода логики $L$ тогда и только тогда, когда $r_{1}^{*}, \ldots, r_{k}^{*}$ есть базис квазитождеств $\mathfrak{F}_{w}(L)$.

Модель Крипке $\langle F, R, V\rangle$, где $V: P_{n} \rightarrow 2^{F}$ и $P_{n}=\left\{p_{1}, p_{2}, \ldots, p_{n}\right\}$, называется $n$-характеристической для логики $L$, тогда и только тогда, когда для любой формулы $\alpha\left(p_{1}, \ldots, p_{n}\right)$ от переменных $p_{1}, \ldots, p_{n}, \alpha \in L \Longleftrightarrow\langle F, R, V\rangle \vDash \alpha$.

В нашем исследовании существенно будет использоваться строение $n$-характеристической модели для финитно аппроксимируемых логик, расширяющих логику $S 4$, с помощью которой будет описана допустимость правил вывода в этих логиках. Следуя гл. 3 в [1], опишем конструкцию этой модели. Пусть задана финитно аппроксимируемая логика $\lambda$, расширяющая логику $S 4$, и пусть задано множество пропозициональных переменных $P_{n}=\left\{p_{1}, p_{2}, \ldots, p_{n}\right\}$. Первый слой данной модели $S_{1}\left(C_{n}(\lambda)\right)$ состоит из множества попарно неизоморфных как модели сгустков со всевозможными означиваниями $V$ переменных из множества $P_{n}$. Предположим, что $S_{\leqslant m}\left(C_{n}(\lambda)\right)$ уже построен. Слой $S_{m+1}\left(C_{n}(\lambda)\right)$ глубины $m+1$ получим следующим образом. Выберем произвольную антицепь сгустков $\mathscr{X} \subset S_{\leqslant m}\left(C_{n}(\lambda)\right)$, содержащую хотя бы один сгусток глубины $m$ и добавим сгусток $C$ из $S_{1}\left(C_{n}(\lambda)\right)$ как ко-накрытие для антицепи $\mathscr{X}$ при условии:

(i) фрейм $C^{R}=\mathscr{X}^{R} \cup\{C\}$ является $\lambda$-фреймом;

(ii) если $\mathscr{X}=\left\{C_{1}\right\}$, то сгусток $C$ не изоморфен подмодели сгустка $C_{1}$.

Продолжая описанную процедуру, в итоге получим модель $C_{n}(\lambda)$. Свойства полученной модели сформулируем в следующих утверждениях.

Предложение 3 ([1]). Для любой финитно аппроксимируемой логики $\lambda$, расширяющей $S 4$, модель $C_{n}(\lambda)$ является $n$-характеристической, и каждый элемент данной модели является формульным.

Предложение 4 ([1]). Для любой финитно аппроксимируемой логики $\lambda$, расширяющей $S 4$, правило вывода $r$ допустимо в $\lambda$, если и только если $r$ истинно на фрейме $C_{n}(\lambda)$ для любого п и при любом формульном означивании переменных.

В данном исследовании нам также понадобится редуцированная форма модальных правил вывода. Говорим, что правило $R$ имеет редуцированную форму, если

$$
R=\bigvee_{1 \leqslant j \leqslant m} \varphi_{j} / \square x_{0},
$$


где

$$
\varphi_{j}=\bigwedge_{0 \leqslant i \leqslant k} x_{j}^{a_{j}} \wedge \bigwedge_{0 \leqslant i \leqslant k} \diamond x_{j}^{b_{j}}, \quad a_{j}, b_{j} \in\{0,1\}, \quad x^{0}=x, \quad x^{1}=\neg x .
$$

Для каждого $\varphi_{j}$ определим также множества

$$
\begin{aligned}
& \theta_{1}\left(\varphi_{j}\right)=\left\{x_{j}: 0 \leqslant j \leqslant k, a_{j}=0\right\}, \\
& \theta_{2}\left(\varphi_{j}\right)=\left\{x_{j}: 0 \leqslant j \leqslant k, b_{j}=0\right\} .
\end{aligned}
$$

Предложение 5 ([1]). Для любого модального правила вывода $R$ существует правило $\operatorname{rf}(R)$ в редуцированной форме, эквивалентное $R$ относительно истинности на $S 4$-алгебрах и $S 4$-фреймах; и $R$ и $\mathrm{rf}(R)$ одновременно выводимы или допустимы в любой модальной логике, расширяющей $S 4$.

Предложение 6 ([11]). Пусть конечно порожденный фрейм Ғ адекватен логике $\lambda \supseteq S 4$, и пусть первый слой данного фрейма содержит хотя бы один одноэлементный сгусток. Тогда если алгебра $\mathscr{F}^{+}$не принадлежит квазимногообразию $\mathfrak{F}_{w}^{Q}(\lambda)$, порожденному свободной алгеброй счетного ранга $\mathfrak{F}_{w}(\lambda)$, то во фрейме $\mathscr{F}$ существует нетривиальная антищепь сгустков, не имеющая $\lambda$-ко-накрытия в $\mathscr{F}, u$ достижимая по отнотению $R$ из некоторого элемента $y \in \mathscr{F}$.

Говорим, что логика $\lambda$, расширяющая логику $S 4$, имеет слабое свойство ко-накрытий, если для любого конечного корневого $\lambda$-фрейма $\mathscr{F}$ и произвольной нетривиальной антицепи $\mathscr{L}$ сгустков из $\mathscr{F}$ фрейм $\mathscr{F}_{1}$, полученный добавлением как корня одноэлементного рефлексивного ко-накрытия к фрейму $\bigcup_{c \in \mathscr{2} R} c^{R}$, также является $\lambda$-фреймом.

Пусть задана модальная логика $\lambda \supseteq S 4$, удовлетворяющая следующим свойствам:

- она финитно аппроксимируема;

- она имеет слабое свойство ко-накрытий;

- она обладает дизъюнктивным свойством.

Для всех чисел $n>1, m \geqslant 1, n, m \in \mathbf{N}$, определим формулы

$$
\begin{aligned}
A_{n} & :=\bigwedge_{1 \leqslant i \leqslant n} \diamond p_{i}, \quad A_{n, m}:=\square\left[\bigwedge_{1 \leqslant i \leqslant n}\left(p_{i} \rightarrow \bigwedge_{1 \leqslant j \leqslant m} \neg \diamond q_{j}\right)\right], \\
B_{m} & :=\bigvee_{\mathscr{D} \subseteq\{1, \ldots, m\}}\left[\bigwedge_{i \in \mathscr{D}} q_{i} \wedge \bigwedge_{i \notin \mathscr{D}, 1 \leqslant j \leqslant m} \neg \diamond q_{i}\right]
\end{aligned}
$$

а также правила вывода

$$
R_{n, m}=\frac{\square\left(A_{n, m} \wedge \neg\left(A_{n} \wedge B_{m}\right)\right) \vee \square z}{\square \neg A_{n} \vee \square z} .
$$

В статье [16] В. В. Рыбаковым была доказана следующая теорема, доказательство которой мы воспроизведем, так как будем использовать его элементы в дальнейшем.

Теорема 1. Правила $R_{n, m}$ допустимь в любой финитно аппроксимируемой логике $\lambda$, расширяющей $S 4$, имеющей слабое свойство ко-накрытий и дизъюнктивное свойство. 
Доказательство. Предположим, что правило вывода $R_{n, m}$ не допустимо в логике $\lambda$. Так как логика $\lambda$ имеет дизъюнктивное свойство, правило

$$
R:=\frac{\square\left(A_{n, m} \wedge \neg\left(A_{n} \wedge B_{m}\right)\right)}{\square \neg A_{n}}
$$

также недопустимо в логике $\lambda$. Тогда по предложению 4 существует формульное означивание $V$ переменных правила $R$, опровергающее $R$ на некоторой $k$-характеристической модели $C_{k}(\lambda)$. Итак, справедливо

$$
C_{k}(\lambda) \vDash_{V} \square\left(A_{n, m} \wedge \neg\left(A_{n} \wedge B_{m}\right)\right) \& C_{k}(\lambda) \not \nvdash_{V} \square \neg A_{n}
$$

Следовательно, существует элемент $a \in C_{k}(\lambda)$ такой, что $a \not \not_{V} \square \neg A_{n}$. Тогда найдутся элементы $b_{1}, \ldots, b_{n} \in C_{k}(\lambda)$ такие, что $a R b_{i} \& b_{i} \vDash_{V} p_{i}$. По слабому свойству конакрытий, существует рефлексивный элемент $b \in C_{k}(\lambda)$, являющийся ко-накрытием для множества $\left\{b_{1}, \ldots, b_{n}\right\}$ :

$$
\{b\}^{R}:=\{b\} \cup \bigcup_{1 \leqslant i \leqslant n}\left(b_{i}\right)^{R} .
$$

По (*), $b \vDash_{V} A_{n, m}$, и по выбору элемента $b \vDash_{V} A_{n}$. Рассмотрим множество

$$
D:=\left\{q_{j} \mid b \vDash_{V} q_{j}\right\}
$$

Так как $b$ является ко-накрытием для $\left\{b_{1}, \ldots, b_{n}\right\}$, легко проверить, что дизъюнктивный член $B_{m}$, соответствующий такому $D$, выполняется на элементе $b$ при означивании $V$. Таким образом, получаем $b \vDash_{V} A_{n} \wedge B_{m}$, что противоречит $b \vDash_{V} \square \neg\left(A_{n} \wedge B_{m}\right)$ по предположению (*).

Теорема доказана.

\section{3. Основной результат}

Пусть задана модальная логика $\lambda \supseteq S 4.1$, удовлетворяющая следующим условиям:

(i) логика финитно аппроксимируема;

(ii) она имеет слабое свойство ко-накрытий;

(iii) она обладает дизъюнктивным свойством.

Напомним, что логика $S 4.1$ определяется как

$$
S 4.1=\mathrm{S} 4 \oplus \square \diamond p \rightarrow \diamond \square p
$$

Соответственно, класс фреймов, адекватных логике $S 4.1$, удовлетворяет условию

$$
\forall x \exists y[x R y \& \forall z(y R z \Rightarrow z=y)]
$$

(см. например, теорему 2.3.49 в [1]). В данном случае все сгустки первого слоя $S 4.1$-фреймов одноэлементны, значит, предложение 6 выполняется для фреймов, адекватных логике $S 4.1$ или ее расширений. 
Для всех чисел $n>1, n \in \mathbf{N}$, определим формулы:

$$
\begin{aligned}
A_{n} & :=\bigwedge_{1 \leqslant i \leqslant n} \diamond p_{i}, \quad A_{n, 1}:=\square\left[\bigwedge_{1 \leqslant i \leqslant n}\left(p_{i} \rightarrow \neg \diamond q\right)\right], \\
B & :=q \vee \neg \diamond q,
\end{aligned}
$$

определим также множество правил вывода

$$
\mathscr{R}_{n}=\frac{\square\left(A_{n, 1} \wedge \neg\left(A_{n} \wedge B\right)\right) \vee \square z}{\square \neg A_{n} \vee \square z} .
$$

Покажем, что последовательность правил вывода $\mathscr{R}_{n}, n>1, n \in \mathbf{N}$, является базисом для допустимых правил вывода логики $\lambda \supseteq S 4.1$, удовлетворяющей условиям (i)-(iii).

Теорема 2. Правила $\mathscr{R}_{n}$ допустимы в любой финитно аппроксимируемой логике $\lambda$, расширяющей $S 4$, имеющей слабое свойство ко-накрытий и дизъюнктивное свойство.

Это следует непосредственно из теоремы 1 , так как правила $\mathscr{R}_{n}$ являются частным случаем правил $R_{n, m}$ при $m=1$.

Пусть задана модальная алгебра $\mathscr{A}:=\mathscr{F}^{+}(X) \in \operatorname{Var}(S 4.1)$, порожденная множеством подмножеств $X \subseteq \mathscr{F}$ обертывающей алгебры $\mathscr{F}^{+}$, где $\mathscr{F}-$ заданный рефлексивный и транзитивный $\lambda$-фрейм, в котором все сгустки глубины 1 одноэлементны. Пусть $\operatorname{rf}(r)-$ правило вывода в редуцированной форме.

Теорема 3. Если правило $\operatorname{rf}(r)$ допустимо в логике $\lambda$, имеет $k$ переменных и опровергается на алгебре $\mathcal{A}$, тогда для некоторого $n>1$ правило $\mathscr{R}_{n}$ также опровергается на $A$.

Доказательство. Наше правило $\operatorname{rf}(r)$ имеет вид

$$
\operatorname{rf}(r)=\bigvee_{1 \leqslant j \leqslant t} \frac{\varphi_{j}}{\square x_{0}},
$$

где

$$
\varphi_{j}:=\bigwedge_{0 \leqslant i \leqslant k} x_{j}^{a} \wedge \bigwedge_{0 \leqslant i \leqslant k} \diamond x_{j}^{b},
$$

$a, b \in\{0,1\}, x^{0}:=x, x^{1}:=\neg x$. Так как $\operatorname{rf}(r)$ опровергается на алгебре $\mathscr{A} \in \operatorname{Var}(L)$, при некотором означивании $V\left(x_{i}\right):=\mathscr{Y}_{i} \in \mathscr{A}$, то

$$
\mathscr{F} \Vdash_{V} \bigvee \varphi_{j} ; \quad \exists b \in \mathscr{F}: b \nVdash_{V} \square x_{0} .
$$

В частности, алгебра $\mathscr{A}$ является конечно порожденной. Соответственно, фрейм $\mathscr{F}$ также можем считать конечно порожденным. Среди всех таких элементов, на которых опровергается заключение правила, выберем элемент $b$ такой, чтобы множество дизъюнктов

$$
\varphi(b):=\left\{\varphi_{j} \mid \exists c \in b^{R}: c \vDash_{V} \varphi_{j}\right\}
$$

было максимальным. Тогда $b^{R} \not \models_{V} \operatorname{rf}(r)$, то есть

$$
\forall c \in b^{R} \quad c \vDash_{V} \bigvee \varphi_{j} ; \quad b \nvdash_{V} \square x_{0}
$$


Рассмотрим алгебру $\left(b^{R}\right)^{+}$, порожденную фреймом $b^{R}$, и ее подалгебру

$$
\mathscr{B}:=\left(b^{R}\right)^{+}\left(V\left(x_{0}\right), V\left(x_{1}\right), \ldots, V\left(x_{k}\right)\right),
$$

порожденную множеством элементов $V\left(x_{0}\right), V\left(x_{1}\right), \ldots, V\left(x_{k}\right)$. Так как наше правило $\operatorname{rf}(r)$ опровергается на $b^{R}$, то $\mathscr{B} \not \nvdash_{V} \operatorname{rf}(r)$. Следовательно, алгебра $\mathscr{B}$ не принадлежит квазимногообразию $\mathfrak{F} \underset{w}{Q}$, порожденному свободной алгеброй счетного ранга $\mathfrak{F} w$ из многообразия $\operatorname{Var}(\lambda)$. В противном случае на этой алгебре истинны все квазитождества, соответствующие допустимым в логике правилам вывода, в том числе и квазитождество, соответствующее правилу $\mathrm{rf}(r)$, получаем противоречие.

Тогда, по предложению 6 , существует нетривиальная антицепь сгустков $X \subset b^{R}$, не имеющая $\lambda$-ко-накрытия $\varepsilon$ в $\mathscr{F}$ (и значит в $b^{R}$ ), порождающего как корень $\lambda$-фрейм. Зафиксируем эту нетривиальную антицепь $X \subset b^{R}$.

Рассмотрим множество $\mathscr{E}$, состоящее из всех дизъюнктивных членов посылки правила $\operatorname{rf}(r)$, имеющих в $\mathscr{B}$ непустое множество истинности, то есть

$$
\mathscr{L}:=\left\{\varphi_{j} \mid \exists c \in b^{R}: c \vDash_{V} \varphi_{j}\right\} .
$$

Определим также множество $\mathscr{D}$ дизъюнктов правила $\operatorname{rf}(r)$, истинных на элементах антицепи $X \subset b^{R}$, то есть

$$
\mathscr{D}:=\left\{\varphi_{s} \mid \exists e \in X e \vDash_{V} \varphi_{s}\right\} .
$$

Предположим, что на $\lambda$-ко-накрытии $\varepsilon$ антицепи сгустков $X \subset b^{R}$ (если бы такое существовало в $\mathscr{F})$ истинна некоторая формула $\varphi_{\varepsilon}$ из множества дизьюнктивных членов посылки правила $\operatorname{rf}(r)$. Непосредственной проверкой можно убедиться, что только для такой формулы $\varphi_{\varepsilon}$ выполняется равенство

$$
\theta_{2}\left(\varphi_{\varepsilon}\right)=\theta_{1}\left(\varphi_{\varepsilon}\right) \cup \bigcup_{\varphi \in \mathscr{D}}\left(\theta_{1}(\varphi) \cup \theta_{2}(\varphi)\right) .
$$

Так как зафиксированная антицепь $X \subset b^{R}$ не имеет ко-накрытия $\varepsilon$ в $\mathscr{F}$ (и значит в $b^{R}$ ), то для всех $\varphi_{j} \in \mathscr{L}$

$$
\theta_{2}\left(\varphi_{j}\right) \neq \theta_{1}\left(\varphi_{j}\right) \cup \bigcup_{\varphi \in \mathscr{D}}\left(\theta_{1}(\varphi) \cup \theta_{2}(\varphi)\right) .
$$

В частности, вследствие нетривиальности антицепи $X \subset b^{R}$, получаем, что $|\mathscr{D}|>1$. Положим также

$$
\begin{aligned}
& P_{V}:=\operatorname{Var}(\operatorname{rf}(r))=\left\{x_{0}, \ldots, x_{k}\right\}, \\
& P_{T}:=\operatorname{Var}\left(\bigcup_{\varphi \in \mathscr{D}}\left(\theta_{1}(\varphi) \cup \theta_{2}(\varphi)\right)\right)=\left\{p \mid \exists c \in X: c \vDash_{V} p \vee c \vDash_{V} \diamond p\right\},
\end{aligned}
$$

и пусть

$$
n:=|\mathscr{D}|, \quad m:=\left|P_{V}-P_{T}\right| .
$$

Ясно, что $n>1, m>0$. Если $m=0$, то есть

$$
\operatorname{Var}\left(\bigcup_{\varphi \in \mathscr{D}}\left(\theta_{1}(\varphi) \cup \theta_{2}(\varphi)\right)\right)=\left\{x_{0}, \ldots, x_{k}\right\},
$$


и $b \vDash_{V} \varphi_{b}$ для некоторого $\varphi_{b} \in \mathscr{L}$ и $b R x$ для всех $x \in X$, то

$$
b \vDash_{V} \diamond x_{i}, \quad 0 \leqslant i \leqslant k .
$$

Тогда

$$
\theta_{2}\left(\varphi_{b}\right)=\theta_{1}\left(\varphi_{b}\right) \cup \bigcup_{\varphi \in \mathscr{D}}\left(\theta_{1}(\varphi) \cup \theta_{2}(\varphi)\right),
$$

что противоречит (1). Следовательно, $m>0$.

Зафиксируем взаимно однозначное соответствие $f$ между $\left\{p_{1}, p_{2}, \ldots, p_{n}\right\}$ и $\mathscr{D}$, а также отображение $g$ множества $P_{V}-P_{T}$ на $\{q\}$. Теперь расширим означивание $V$ на $\mathscr{B}$ с переменных правила $\operatorname{rf}(r)$ на переменные правила $\mathscr{R}_{n}$ следующим образом:

$$
V\left(p_{i}\right):=V\left(f\left(p_{i}\right)\right) \quad \& \quad V(q):=V\left(P_{V}-P_{T}\right) .
$$

Легко заметить, что

$$
\begin{aligned}
& x \vDash_{V} p_{i} \Longleftrightarrow x \vDash_{V} \varphi_{j_{i}}, \quad \varphi_{j_{i}} \in \mathscr{D}, \\
& x \vDash_{V} q \Longleftrightarrow x \vDash_{V} y, \quad y \in \operatorname{Var}(\operatorname{rf}(r)) \backslash P_{T} .
\end{aligned}
$$

Покажем, что при таком определении означивания $V$ правило $\mathscr{R}_{n}$ опровергается на алгебре $\mathscr{B}$.

Предложение 7. Верно, что

$$
b \vDash_{V} A_{n}, \quad b \nvdash_{V} \square \neg A_{n} .
$$

Доказательство. Первая часть легко следует из определения алгебры $\mathscr{B}$ и выбора множеств $\mathscr{D}$ и $\mathscr{L}$. Действительно, для всех $e \in X$

$$
b \operatorname{Re} \wedge e \vDash_{V} \varphi_{j}, \quad \varphi_{j} \in \mathscr{L} .
$$

Следовательно, по определению означивания выполняется

$$
e \vDash_{V} p_{i}, \quad 1 \leqslant i \leqslant n .
$$

Тогда по транзитивности отношения заключаем, что

$$
b \vDash_{V} \diamond p_{i}
$$

для всех $i \leqslant n$, и значит,

$$
b \vDash_{V} \bigwedge_{1 \leqslant i \leqslant n} \diamond p_{i}
$$

Вторая часть следует из рефлексивности элемента $b$ : если $b \vDash_{V} \square \neg A_{n}$, то на всех элементах, достижимых из $b$, в том числе и на самом $b$, должно быть истинно $\neg A_{n}$, что противоречит $b \vDash_{V} A_{n}$. Утверждение доказано.

Предложение 8. Справедливо соотношение

$$
b \vDash_{V} \square A_{n, 1} .
$$


Доказательство. Возьмем произвольное $c \in b^{R}$ и предположим, что $c \vDash_{V} p_{i}$ для всех $i$, отсюда следует, что $c \vDash_{V} f\left(p_{i}\right)$, и значит, $c \vDash_{V} \varphi_{j}$ для всех $\varphi_{j} \in \mathscr{D}$. Тогда существует $y \in P_{T}: c \vDash_{V} y \vee \diamond y$, что влечет соотношение $\forall x \in\left[P_{V}-P_{T}\right] c \vDash_{V} \neg \diamond x$. Следовательно, $c \vDash_{V} \neg \diamond q$. Таким образом, $c \vDash_{V} A_{n, 1}$, откуда в силу произвольности выбора элемента $c \in b^{R}$ заключаем, что $b \vDash_{V} \square A_{n, 1}$. Предложение доказано.

Предложение 9. Справедливо соотношение

$$
b \not \models_{V} \square\left(A_{n} \wedge B\right) .
$$

Доказательство. Предположим, что $b \vDash_{V} \square A_{n}$, то есть для всех $c \in b^{R}$ выполняется соотношение $c \vDash_{V} A_{n}$. Кроме того, в силу выбора элемента $c$, выполняется соотношение $c \vDash_{V} \varphi_{j}, \varphi_{j} \in \mathscr{L}$. Тогда

$$
c \vDash_{V} A_{n} \Longleftrightarrow c \vDash_{V} \bigwedge_{1 \leqslant i \leqslant n} p_{i} \Longleftrightarrow c \vDash_{V} \varphi_{j_{i}},
$$

для всех $i=[1, n]$ и $\varphi_{j_{i}} \in \mathscr{D}$. Следовательно, в силу рефлексивности элемента $c$, заключаем, что $c \vDash_{V} \diamond \varphi_{j_{i}}, \varphi_{j_{i}} \in \mathscr{D}$. Отсюда вытекает, что $P_{T} \subseteq \theta_{2}\left(\varphi_{j}\right)$.

Пусть также

$$
c \vDash_{V} q \vee \neg \diamond q, \quad c \vDash_{V} \diamond x_{i}, \quad x_{i} \in P_{V}-P_{T} .
$$

Следовательно, в соответствии с истинностью $c \vDash_{V} B$, получаем, что $c \vDash_{V} x_{i}$ для $x_{i} \notin P_{T}$. Таким образом, $x_{i} \in \theta_{1}\left(\varphi_{j}\right)$.

Итак, совместно доказанное выше влечет, что

$$
\theta_{2}\left(\varphi_{j}\right)=\theta_{1}\left(\varphi_{j}\right) \cup \bigcup_{\varphi \in \mathscr{D}}\left(\theta_{1}(\varphi) \cup \theta_{2}(\varphi)\right),
$$

и $\varphi_{j} \in \mathscr{L}$, что противоречит (1). Предложение доказано.

Из предложений 7-9 следует, что справедлива следующая лемма.

Лемма 1. Справедливо соотношение

$$
\vDash_{V} \square\left(A_{n, 1} \wedge \neg\left(A_{n} \wedge B\right)\right) \quad \& \quad \mathscr{B} \not \nvdash_{V} \square \neg A_{n} .
$$

Теперь мы должны задать означивание $V$ переменной $z$ правила $\mathscr{R}_{n}$ так, чтобы опровергнуть $\mathscr{R}_{n}$ на алгебре $\mathscr{A}$.

Лемма 2. Справедливо соотношение

$$
\mathscr{A} \not \models_{V} \mathscr{R}_{n}
$$

Доказательство. Так как $b \vDash_{V} \bigvee \varphi_{j}$, существует единственная формула $\varphi_{b} \in \mathscr{Z}$ такая, что $b \vDash_{V} \varphi_{b}$. Определим означивание $V$ переменной $z$ на алгебре $\mathscr{A}$ следующим образом:

$$
V(z):=V\left(\neg\left[\diamond \varphi_{b} \wedge \bigwedge_{\varphi_{j} \in \varphi(b)} \diamond \varphi_{j} \wedge \square\left(\bigvee_{\varphi_{j} \in \mathscr{L}} \varphi_{j}\right)\right]\right) .
$$

Так как $b \vDash_{V} \varphi_{b}$ и

$$
\mathscr{Z}=\left\{\varphi_{j} \mid \exists c \in b^{R} c \vDash_{V} \varphi_{j}\right\},
$$


то $b \not \nvdash_{V} z$. Следовательно, $b \not \nvdash_{V} \square z$, и, как было показано ранее, $b \not \nvdash_{V} \square \neg A_{n}$. Таким образом, заключение правила $\mathscr{R}_{n}$ опровергается на элементе $b \in \mathscr{A}$ при означивании $V$.

Покажем, что на алгебре $\mathscr{A}$ посылка правила $\mathscr{R}_{n}$ истинна при означивании $V$. Пусть существует $c \in \mathscr{F}: c \not_{V} \square z$. Тогда по определению $V(z)$ получаем, что

$$
\exists d: c R d \wedge d \vDash_{V} \varphi_{b} \wedge c \vDash_{V} \bigwedge_{\varphi_{j} \in \varphi(b)} \diamond \varphi_{j} \wedge \square\left(\bigvee_{\varphi_{j} \in \mathscr{I}} \varphi_{j}\right)
$$

В силу выбора элемента $b, b \not \models_{V} \square x_{0}$. Отсюда заключаем, что существует элемент $g$, достижимый по отношению $R$ из элемента $b$, такой, что выполняется соотношение $g \nvdash_{V} x_{0}$, в частности, $g \vDash_{V} \neg x_{0}$. В силу истинности на $g \in b^{R}$ посылки правила $\mathscr{R}_{n}$ по лемме1 можем заключить, что $g \vDash_{V} \varphi_{j}$ для некоторого $\varphi_{j} \in \mathscr{L}$. Отсюда в силу (2) $\exists w \in c^{R}: w \vDash_{V} \varphi_{j}$ для того же $\varphi_{j} \in \mathscr{L}$. Следовательно, $w \not_{V} x_{0}$, откуда вытекает, что $c \not \models_{V} \square x_{0}$.

Напомним, что множество $\varphi(b)$ выбиралось как максимальное множество дизъюнктов $\varphi_{j}$, истинных на $b^{R}$. В силу (2), множество $\varphi(c)$ также является максимальным и $\varphi(c)=\varphi(b)$. Таким образом, множество элементов, достижимых из элементов $b$ и $c$ соответственно, имеют в совокупности одно и то же множество формул $\varphi_{j}$, истинных при означивании $V$. Отсюда и из соотношения $c \not \nvdash_{V} \square x_{0}$ заключаем, что

$$
c \vDash_{V} \square\left(A_{n, 1} \wedge \neg\left(A_{n} \wedge B\right)\right),
$$

при этом используются те же аргументы, что и для элемента $b$ при доказательстве леммы 1. Таким образом, посылка правила истинна на произвольном элементе $c \in \mathcal{A}$, и теорема 3 доказана.

Из теорем 2 и 3 непосредственно вытекает следующее утверждение.

Теорема 4. Пусть задана модальная логика $\lambda \supseteq S 4.1$ такая, что она

(1) финитно аппроксимируема;

(2) имеет слабое свойство ко-накрытий;

(3) обладает дизъюнктивным свойством.

Тогда множество правил $\left\{\mathscr{R}_{n}, n>1, n \in \mathbf{N}\right\}$, образует базис допустимых правил вывода логики $\lambda$.

Следствие 1. Множество правил $\left\{\mathscr{P}_{n}, n>1, n \in \mathbf{N}\right\}$ образует базис допустимых правил вывода логики S4.1, Grz.

Говорим, что логика $\lambda$ конечной ширины $\mathscr{L}$, расширяющая логику $S 4$, имеет слабое свойство $\mathscr{L}$-ко-накрытий, если для любого конечного корневого $\lambda$-фрейма $\mathscr{F}$ и произвольной нетривиальной антицепи $\mathscr{X}$ сгустков из $\mathscr{F}$, состоящей не более чем из $\mathscr{L}$ сгустков, фрейм $\mathscr{F}_{1}$, полученный добавлением как корня одноэлементного рефлексивного конакрытия к фрейму $\bigcup_{c \in \mathscr{L} R} c^{R}$, также является $\lambda$-фреймом.

Теорема 5. Пусть задана модальная логика $\lambda \supseteq G r z$ такая, что она

(i) финитно аппроксимируема; 
(ii) имеет слабое свойство $\mathscr{L}$-ко-накрытий;

(iii) обладает дизъюнктивным свойством;

(iv) ширина логики конечна и ограничена числом $\mathscr{L}$.

Тогда множество правил $\left\{\mathscr{\Re}_{n}, 1 \leqslant n \leqslant \mathscr{L}\right\}$ образует базис допустимых правил вывода логики $\lambda$. В частности, логика имеет конечный (и независимый) базис для допустимых правил вывода.

Доказательство. Доказательство допустимости правил $\left\{\mathscr{R}_{n}, 1 \leqslant n \leqslant \mathscr{L}\right\}$ повторяет доказательство теоремы 2. Доказательство выводимости произвольного допустимого в логике $\lambda$ правила вывода из этого набора правил $\left\{\mathscr{R}_{n}, n \leqslant \mathscr{L}\right\}$ полностью повторяет доказательство теоремы 4. Конечность базиса $\mathscr{R}_{n}, 1 \leqslant n \leqslant \mathscr{L}$, следует из конечности ширины логики. Так как ширина логики ограничена числом $\mathscr{L}$ и все сгустки одноэлементные, мощность множества $\mathscr{D}$ (антицепи $\mathscr{X}$, допускающей приписывание снизу $\lambda$-ко-накрытие) также ограничена этим числом. По определению,

$$
0<n=|\mathscr{D}| \leqslant \mathscr{L} \text {. }
$$

Лемма 3. Пусть логика $\lambda$ удовлетворяет условиям теоремы 5. Тогда правила $\mathscr{R}_{k}, k>\mathscr{L}$, недопустимы в логике $\lambda$.

Доказательство. Доказательство утверждения проведем следующим образом: построим фрейм $\mathscr{F}$, опровергающий данное правило $\mathscr{R}_{k}$ при некотором означивании, затем с помощью р-морфизма из фрейма $n$-характеристической модели $C_{n}(\lambda)$ на фрейм $\mathscr{F}$ перенесем означивание на $n$-характеристическую модель, откуда будет следовать недопустимость правила в логике $\lambda$ согласно предложению 4.

Определим $\lambda$-фрейм $\mathscr{F}$ следующим образом. Первый слой данного фрейма состоит из единственного рефлексивного элемента $a_{0}$, то есть $S_{1}(\mathscr{F}):=\left\{a_{0}\right\}$. Второй слой этого фрейма образует антицепь из $\mathscr{L}+1$ рефлексивных элементов: $S_{2}(\mathscr{F}):=\left\{a_{1}, a_{2}, \ldots a_{\mathscr{L}+1}\right\}$. Для построения третьего слоя выбираем все нетривиальные антицепи, состоящие не более чем из $\mathscr{L}$ сгустков второго слоя. Затем к каждой такой антицепи приписываем снизу рефлексивный элемент как ко-накрытие. Заметим, что при таком построении антицепь $\left\{a_{1}, a_{2}, \ldots, a_{\mathscr{L}+1}\right\}$ не имеет ко-накрытия.

Пусть $S_{\leqslant k}(\mathscr{F})$ глубины не более $k$ уже построен. Следующий слой $S_{k+1}(\mathscr{F})$ глубины $k+1$ получим следующим образом. Выберем все нетривиальные антицепи сгустков $\mathscr{L} \subset S_{\leqslant k}(\mathscr{F})$, содержащие хотя бы один сгусток глубины $k$ и состоящие не более чем из $\mathscr{L}$ сгустков. Затем к каждой такой антицепи приписываем снизу вырожденный сгусток как ко-накрытие. Продолжая описанную процедуру для последующих слоев, получим $\lambda$ фрейм $\mathscr{F}$ ширины $\mathscr{L}$, в котором любая нетривиальная антицепь, состоящая не более чем из $\mathscr{L}$ сгустков, имеет ко-накрытие.

Определим теперь на фрейме $\mathscr{F}$ означивание $V$ переменных правила $\left\{\mathscr{R}_{k}, k>\mathscr{L}\right\}$ следующим образом:

$$
\begin{aligned}
V(q) & :=S_{3}(\mathscr{F}) ; \\
V\left(p_{1}\right) & :=a_{1}, \quad V\left(p_{2}\right):=a_{2}, \quad \ldots, \quad V\left(p_{\mathscr{L}}\right):=a_{\mathscr{L}}, \\
V\left(p_{\mathscr{L}+1}\right) & :=V\left(p_{\mathscr{L}+2}\right)=\ldots=V\left(p_{k}\right):=a_{\mathscr{L}+1} .
\end{aligned}
$$

Несложно увидеть, что при таком означивании правило $\mathscr{R}_{k}, k>\mathscr{L}$, опровергается на фрейме $\mathscr{F}$. Действительно, по свойству (ii) логики $\lambda$ и по построению фрейма $\mathscr{F}$ существует элемент $b \in \mathscr{F}$ такой, что $b R a_{i}, 1 \leqslant i \leqslant \mathscr{L}+1$, то есть найдется $R$-предшественник 
антицепи $\left\{a_{1}, a_{2}, \ldots, a_{\mathscr{L}+1}\right\}$. Следовательно,

$$
b \vDash_{V} \bigwedge_{1 \leqslant i \leqslant k} \diamond p_{i},
$$

то есть

$$
b \not \nvdash_{V} \square \neg A_{k} .
$$

Далее, для всех $e \in S_{\leqslant 2}(\mathscr{F})$ выполняется соотношение

$$
e \vDash_{V} \neg \diamond q \wedge \neg A_{k},
$$

откуда заключаем, что

$$
e \vDash_{V} \neg\left(A_{k} \wedge B\right)
$$

Очевидно также, что для всех элементов $z \in S_{3}(\mathscr{F})$ по построению справедливо соотношение $z \vDash_{V} \neg A_{k}$ (так как антицепь $\left\{a_{1}, a_{2}, \ldots a_{\mathscr{L}+1}\right\}$ не имеет ко-накрытия), отсюда выводим, что для всех $z \in S_{3}(\mathscr{F})$

$$
z \vDash_{V} \neg\left(A_{k} \wedge B\right) .
$$

Для элементов глубины, большей 3, верно, что

$$
z \vDash_{V} \neg q \wedge \diamond q,
$$

откуда снова следует истинность

$$
z \vDash_{V} \neg\left(A_{k} \wedge B\right)
$$

Непосредственно по определению означивания выполняется также соотношение

$$
z \vDash_{V} \bigwedge_{1 \leqslant i \leqslant k}\left(\neg p_{i} \vee \neg \diamond q\right),
$$

верное для всех $z \in \mathscr{F}$, откуда

$$
z \vDash_{V} A_{k, 1} \text {. }
$$

для всех $z \in \mathscr{F}$. Таким образом, заключаем, что для всех $y \in \mathscr{F}$

$$
y \vDash_{V} A_{k, 1} \wedge \neg\left(A_{k} \wedge B\right) .
$$

В силу произвольности элемента $у$ посылка правила $\mathscr{R}_{k}, k>\mathscr{L}$, истинна на фрейме $\mathscr{F}$, а заключение опровергается на элементе $b$.

Теперь остается заметить, что так как любая нетривиальная антицепь сгустков мощности, не большей $\mathscr{L}$, имеет $\lambda$-ко-накрытие в $\mathscr{F}$, то для некоторого $n$ существует р-морфизм из фрейма $n$-характеристической модели $C_{n}(\lambda)$ на фрейм $\mathscr{F}$. Перенося с помощью этого p-морфизма означивание $V$ на фрейм $C_{n}(\lambda)$, получим р-морфизм моделей, сохраняющий истинность формул. Следовательно, правило $\mathscr{R}_{k}, k>\mathscr{L}$, опровергается на $n$-характеристической модели $C_{n}(\lambda)$, откуда заключаем, что оно не допустимо в логике $\lambda$. 
Покажем теперь, что число финитно аппроксимируемых логик, удовлетворяющим условиям теоремы 5, не менее чем счетно. Для этого определим последовательность $G r z$-фреймов $\mathscr{F}_{i}, i>2, i \in \mathbf{N}$, следующим образом. Зафиксируем произвольное число $K>2$. Первый слой фрейма $\mathscr{F}_{i}$ для каждого $i$ состоит из $i$ одноэлементных сгустков, образующих антицепь. Для построения второго слоя фрейма $\mathscr{F}_{i}$ выбираем все нетривиальные антицепи, содержащие не более чем $K$ сгустков первого слоя, и к каждой такой антицепи приписываем снизу вырожденный сгусток как ко-накрытие. Для построения третьего слоя опять выбираем все нетривиальные антицепи, состоящие не более чем из $K$ сгустков первого и второго слоя, и содержащие хотя бы один сгусток второго слоя. Затем к каждой такой антицепи приписываем снизу вырожденный сгусток как ко-накрытие. Продолжая описанную процедуру для последующих слоев, получим бесконечный фрейм ширины $K$, в котором любая нетривиальная антицепь, состоящая не более чем из $K$ сгустков, имеет ко-накрытие.

Затем определим логики

$$
\lambda_{i}:=\lambda\left(\mathscr{F}_{i}\right), \quad i \in \mathbf{N} .
$$

Ясно, что полученные логики являются финитно аппроксимируемыми и имеют ширину $K$. Из определения фреймов $\mathscr{F}_{i}$ также следует, что логики $\lambda_{i}$ имеют слабое свойство $\mathscr{L}$-ко-накрытий.

Несложно также заметить, что данные логики обладают дизъюнктивным свойством. Действительно, если $\square \alpha \vee \square \beta \in \lambda_{i}$, но $\square \alpha \notin \lambda_{i}$ и $\square \beta \notin \lambda_{i}$, то существуют фреймы $F_{1}, F_{2}$ такие, что $F_{1} \not \models \square \alpha, F_{2} \not \models \square \beta$. Без потери общности можем считать эти фреймы корневыми, то есть $F_{1}=a^{R}, F_{2}=b^{R}$. Рассмотрим фрейм

$$
G:=a^{R} \sqcup b^{R}
$$

Аналогично тому, как мы строили фреймы $\mathscr{F}_{i}$, добавляя по слоям (начиная с первого) ко-накрытия ко всем антицепям, состоящим не более чем из $K$ сгустков и не имеющим ко-накрытия в $G$, получим ко-последователь этого фрейма, адекватного логике $\lambda_{i}$. В полученном фрейме антицепь $\{a, b\}$ по слабому свойству $\mathscr{L}$-ко-накрытий имеет ко-накрытие. Очевидно, что на этом ко-накрытии опровергается формула $\square \alpha \vee \square \beta$, что противоречит предположению $\square \alpha \vee \square \beta \in \lambda_{i}$.

Заметим также, что аналогичным образом можно показать, что условиям теоремы 4 удовлетворяет не менее чем счетное число логик. Для этого можно построить последовательность фреймов $\mathscr{F}_{i}^{w}$ (аналогично тому, как были построены фреймы $\mathscr{F}_{i}$ ), добавляя на каждом шаге построения ко-накрытия ко всем нетривиальным антицепям, а не только состоящим не более чем из $K$ сгустков. Тогда, аналогичным образом можно показать, что логики $\lambda_{i}:=\lambda\left(\mathscr{F}_{i}^{w}\right), i \in \mathbf{N}$, удовлетворяют условиям (i)-(iii) этой теоремы.

Известно, что Т-перевод Геделя-Маккинси-Тарского $\sigma$ расширения $\lambda$ интуиционистской логики Int в наибольший модальный напарник $\sigma(\lambda)$ сохраняет многие важные свойства логики: финитную аппроксимируемость, дизъюнктивное свойство, ширину логики и др. (см. например, теоремы 2.7.2, 2.7.21 в [1]). Кроме того, логика $\lambda \supseteq$ Int и ее наибольший модальный напарник $\sigma(\lambda) \supseteq G r z$ имеют один и тот же характеристический класс фреймов. По теореме 3.2.2 из [1], правило вывода $r$ допустимо в суперинтуиционистской логике $\lambda$, если и только если $T(r)$ допустимо в наибольшем модальном напарнике $\sigma(\lambda)$. Отсюда и теорем 4, 5 вытекает следующая теорема. 
Теорема 6. Пусть задана логика $\lambda \supseteq$ Int, удовлетворяющая условиям (i)-(iii) теоремы 4 или условиям (i)-(iv) теоремы 5. Тогда множество правил $\left\{\mathscr{R}_{n}^{i}: T\left(\mathscr{R}_{n}^{i}\right)=\mathscr{R}_{n}, n>1\right.$, $n \in \mathbf{N}\}$ образует базис допустимых правил вывода логики $\lambda$. В частности, если ширина логики конечна и равна $\mathscr{L}$, то логика $\lambda$ имеет конечный (и независимый) базис $\left\{\mathscr{\Re}_{n}^{i}: T\left(\mathscr{R}_{n}^{i}\right)=\mathscr{R}_{n}, 1<n \leqslant \mathscr{L}\right\}$ для допустимых правил вывода.

Очевидно, что полученные базисы допустимых правил дают также описание базиса квазитождеств, истинных на свободной алгебре счетного ранга $\mathscr{F}_{w}(\lambda), \lambda \supseteq S 4.1$ (Grz, Int) и логика $\lambda$ удовлетворяет условиям теорем 4, 5 или 6 соответственно.

\section{Список литературы}

1. Rybakov V. V., Admissibility of logical inference rules. Elsevier, Amsterdam, 1997.

2. Harrop R., Concerning formulas of the types $A \rightarrow B \vee C, A \rightarrow \exists x B(x)$. J. Symbolic Logic (1960) 25, 27-32.

3. Минц Г. Е., Выводимость допустимых правил. Зап. научн. сем. ЛОМИ (1973) 32, 85-89.

4. Port J., The deducibilities of S5. J. Phylosophical Logic (1981) 10, 409-422.

5. Lorenzen P., Einführung in die operative Logik und Mathematik. Springer, Heidelberg, 1969.

6. Friedman H., One hundred and two problems in mathematical logic. J. Symbolic Logic (1975) 40, 113-130.

7. Рыбаков В. В., Критерий допустимости правил вывода в модальной системе $S 4$ и интуиционистской логики Н. Алгебра и логика (1984) 23, №5, 369-384.

8. Ghilardi S., Unification in intuitionistic logic. J. Symbolic Logic (1999) 64, 859-880.

9. Циткин А. И., О допустимых правилах интуиционистской логики высказываний. Матем. сб. (1977) 102, №2, 314-323.

10. Рыбаков В. В., Базис для допустимых правил логики $S 4$ и интуиционистской логики $H$. Алгебра и логика (1985) 24, №1, 55-68.

11. Римацкий В. В., Базисы допустимых правил вывода табличных модальных логик глубины 2. Алгебра и логика (1996) 35, №6, 612-623.

12. Римацкий В. В., О конечной базируемости по допустимости модальных логик ширины 2. Алгебра и логика (1999) 38, №4, 436-455.

13. Rybakov V., Terziler M., Remazki V., A basis in semi-reduced form for the admissible rules of the intuitionistic logic IPC. Math. Logic Q. (2000) 46, 207-218.

14. Iemhoff R., On the admissible rules of intuitionistic propositional logic. J. Symbolic Logic (2001) 66, 281-294.

15. Iemhoff R., A(nother) characterization of intuitionistic propositional logic. Ann. Pure Appl. Logic (2001) 113, 161-173.

16. Rybakov V. V., Construction of an explicit basis for rules admissible in modal system S4. Math. Logic Q. (2001) 47, 441-451.

17. Wolter F., Zakharyaschev M., Undecidability of the unification and admissibility problems for modal and description logics. ACM TOCL 9, 1-20.

18. Голованов М. И., Юрасова Е. М., Критерий допустимости правил вывода логики с оператором «завтра», Деп. ВИНИТИ, №1654-В2004. Красноярский гос. ун-т, Красноярск, 2004.

19. Rybakov V. V., Logical consecutions in intransitive temporal linear logic of finite intervals. J. Logic Comput. (2005) 15, 633-657. 
20. Rybakov V. V., Logical consecutions in discrete linear temporal logic. J. Symbolic Logic (2005) 70, 1137-1149.

21. Rybakov V. V., Branching time logics $\mathscr{B} \mathscr{\mathscr { L }} \mathcal{N}_{\mathrm{N}, \mathrm{N}^{-1}}^{\mathrm{U}, \mathrm{S}}(\mathscr{E})_{\alpha}$ with operations Until and Since based on bundles of integer numbers, logical consecutions, deciding algorithms. Theory Comput. Syst. (2008) 43, 254-271.

22. Golovanov M. I., Kosheleva A. V., Rybakov V. V., Logic of visibility, perception, and knowledge and admissible inference rules. Logic J. IGPL (2005) 13, 201-209.

23. Calardo E., Rybakov V. V., An axiomatisation for the multi-modal logic of knowledge and linear time LTK. Logic J. IGPL (2007) 15, 239-254.

Статья поступила 7.06.2007. 